\title{
Hyperhomocystinemia in patients with coronary artery disease
}

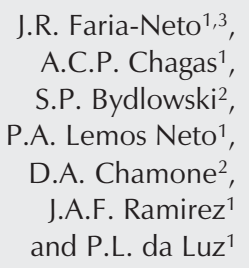

\author{
${ }^{1}$ Instituto do Coração, ${ }^{2}$ Laboratório de Hematologia Molecular (LIM-31), \\ Disciplina de Hematologia e Hemoterapia, Faculdade de Medicina, \\ Universidade de São Paulo, São Paulo, SP, Brasil \\ ${ }^{3}$ Pontifícia Universidade Católica do Paraná, Curitiba, PR, Brasil
}

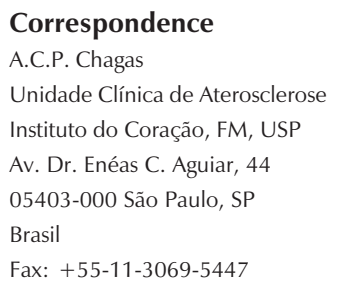

Received June 21, 2004 Accepted November 28, 2005

\begin{abstract}
Hyperhomocystinemia has been related to an increased risk of cardiovascular disease in several studies. The C677T polymorphism for the gene that encodes the methylenetetrahydrofolate reductase enzyme (MTHFR) and low plasma folate levels are common causes of hyperhomocystinemia. Due to differences in nutritional patterns and genetic background among different countries, we evaluated the role of hyperhomocystinemia as a coronary artery disease (CAD) risk factor in a Brazilian population. The relation between homocysteine (Hcy) and the extent of $\mathrm{CAD}$, measured by an angiographic score, was determined. A total of 236 patients referred for coronary angiography for clinical reasons were included. CAD was found in $148(62.7 \%)$ patients and 88 subjects had normal or near normal arteries. Patients with CAD had higher Hcy levels [mean (SD)] than those without disease (14 (6.8) vs 12.5 (4.0) $\mu \mathrm{M} ; \mathrm{P}=0.04)$. Hyperhomocystinemia (Hcy $>17.8 \mu \mathrm{M})$ prevalence was higher in the CAD group: $31.1 \mathrm{vs}$ $12.2 \%(\mathrm{P}=0.01)$. After adjustment for major risk factors, we found an independent association between hyperhomocystinemia and CAD $(\mathrm{OR}=2.48 ; 95 \% \mathrm{CI}=1.02-6.14)$. Patients with a more advanced coronary score had a higher frequency of hyperhomocystinemia and tended to have higher mean Hcy levels. An inverse relation between plasma folate and Hcy levels was found $(\mathrm{r}=-0.14 ; \mathrm{P}=0.04)$. Individuals with the MTHFR C677T polymorphism had a higher prevalence of hyperhomocystinemia than those without the mutated allele. We conclude that hyperhomocystinemia is independently associated with CAD, with a positive association between Hcy level and disease severity.
\end{abstract}

\section{Introduction}

Atherosclerosis is the leading cause of mortality in the Western world. In most patients, traditional risk factors such as dyslipidemia, hypertension, smoking, and diabetes can be identified. The reduction of cardio-

\section{Key words}

- Hyperhomocystinemia

- Homocysteine

- Methylenetetrahydrofolate reductase

- Atherosclerosis

- Folic acid deficiency vascular events can be achieved by controlling these factors. Nevertheless, the prevention of disease progression and new events in patients with established coronary artery disease (CAD) and no "known" risk factors is still a great challenge. Therefore, there is a continuous search for new risk factors that 
could explain the disease in these patients; hyperhomocystinemia is one of them.

Homocysteine (Hcy) is a sulfhydryl amino acid involved in methionine metabolism. The detrimental effect of severe hyperhomocystinemia on the cardiovascular system was first described by McCully (1) in 1969. He reported diffuse atherosclerotic lesions in a post-mortem study of two children with homocystinuria, an inborn error of Hcy metabolism in which extremely high plasma Hcy levels are found. Fatal events before the age of 30 occur in $25 \%$ of these patients (2).

The harmful effect of hyperhomocystinemia on the vascular system has been confirmed by experimental studies in animals (3). After the introduction of reliable routine methods for Hcy determination, clinical studies showed the association of mild to moderate hyperhomocystinemia not only with coronary disease (4-6), but also with stroke (7) and peripheral artery disease (8).

Plasma Hcy concentration is dependent on some nutritional and genetic factors. A low plasma folate level is a common cause of hyperhomocystinemia, but vitamin $B_{12}$ deficiency may also play a role. Folate is a substrate for the remethylation cycle of Hcy, and vitamin $B_{12}$ is a co-factor in its metabolism. Folate supplementation is an effective therapy for Hcy normalization (9).

A common polymorphism for the enzyme 5,10-methylenetetrahydrofolate reductase (MTHFR) is related to mild to moderate elevation of plasma Hcy levels. This enzyme converts 5,10-methylenetetrahydrofolate to 5-methyltetrahydrofolate, required for the conversion of Hcy to methionine. The C677T polymorphism results in an amino acid change from alanine to valine. This substitution causes thermolability and reduction of enzyme activity (10).

Since genetic and nutritional differences are present among different countries, it is reasonable to assume that Hcy may play a different role as a coronary risk factor ac- cording to the population studied. To the best of our knowledge, no study assessed the relation between Hcy and coronary disease in any Latin American country, a developing region susceptible to nutritional deficiencies (11). Although the prevalence of malnutrition is decreasing in Brazil, some diseases like anemia, highly dependent on iron, folate and vitamin $\mathrm{B}_{12}$ intake are now reaching epidemic levels (12).

The aim of the present cross-sectional study was to evaluate the role of hyperhomocystinemia as a coronary risk factor in a Brazilian population. Moreover, using an angiographic index, we evaluated the association between plasma Hcy level and the extent of coronary disease.

\section{Patients and Methods}

\section{Patients}

On the assumption that up to $25 \%$ of patients would have normal or near normal arteries upon an angiogram, sample size was calculated with $\alpha=0.05$ and a power of $90 \%$ to detect a $16 \%$ difference in hyperhomocystinemia prevalence. So, 236 patients referred to the Heart Institute for a selective coronary angiogram were enrolled. All patients had been previously seen by their attending physician, and the angiography was requested based on the clinical judgment of the latter. Patients whose coronary angiogram was requested for a reason other than suspected coronary disease were also included. We excluded patients younger than 18 years, with acute coronary syndrome in the last 30 days, those who had taken any medication containing vitamins or folic acid in the 6 weeks before enrollment, and patients with creatinine above $1.5 \mathrm{mg} / \mathrm{dL}$.

A questionnaire with clinical data was completed by all patients on the day of enrollment. Blood pressure, weight and height were recorded. All measurements were performed by the same research nurse, using 
the same technique and always the same calibrated equipment. The study was approved by the institutional Ethics Committee and written informed consent was obtained from all patients.

\section{Coronary angiography}

Angiograms were evaluated by an experienced angiographer blind to the clinical data. CAD was considered to be present when any stenotic lesion $\geq 40 \%$ in any of major epicardial coronaries and their branches was observed. Subjects with normal or nearnormal arteries (no lesion greater than $40 \%$ ) formed a control group.

All angiograms were scored according to the Friesinger index (13). This index ranges from 0 (completely normal arteries) to 15 . Each of the 3 arteries (right coronary, circumflex and anterior descending artery), with their major branches, was analyzed independently and scored from 0 to 5 . The final score was the sum of the results for each artery. An artery with no wall irregularity was scored as 0 . Score 1 was determined by parietal irregularities, less than $30 \%$. If the artery had a single stenotic lesion causing a narrowing of less than $70 \%$, the score was 2 . The same degree of obstruction, but at more than one specific site of the artery, was scored as 3 . An artery with any lesion greater than $70 \%$ was scored as 4 . A score of 5 was assigned when complete occlusion of the proximal right coronary, circumflex or anterior descending artery was found. Lesions in the left main coronary were assessed using the same scoring system, but doubled (the lesion was considered in two arteries).

\section{Measurement of plasma homocysteine}

Blood samples were collected before angiography, after a 12-h fast. The samples were kept on ice and immediately centrifuged at $0^{\circ} \mathrm{C}$ to obtain plasma. Plasma Hcy levels were quantified by high-performance liquid chromatography using a Shimadzu Class-Vp System. The technique described by Fiskerstrand et al. (14) was used.

\section{Genetic analysis}

Genotyping for the C677T polymorphism of MTHFR was performed by PCR amplification. The reaction primers have been described elsewhere (15). The resulting fragments were separated by $1.5 \%$ agarose gel electrophoresis.

\section{Definitions of risk factors}

Age was evaluated as a continuous and categorical variable. Hypertension was defined as a history of systolic blood pressure $>140 \mathrm{mmHg}$ and/or diastolic pressure $>90$ $\mathrm{mmHg}$, confirmed by measurement at enrollment, or antihypertensive therapy. Patients were considered to be smokers when regularly using tobacco for the last 6 months. Diabetes was defined as fasting glucose above $126 \mathrm{mg} / \mathrm{dL}$ or use of hypoglycemic drug therapy. Hyperlipidemia was present if total cholesterol $>240 \mathrm{mg} / \mathrm{dL}$, or LDL-cholesterol $>160 \mathrm{mg} / \mathrm{dL}$ or if the patient was on lipid-lowering therapy.

Hyperhomocystinemia was defined according to values found in patients with normal or near-normal coronary arteries. We defined hyperhomocystinemia as any value above the 90th percentile for this group.

\section{Statistical analysis}

Continuous variables are reported as means \pm SD. Categorical variables are presented as percentages. Log transformation was used for analysis of skewed data (Hcy). Univariate (2-sided $t$-tests and chi-square tests, Pearson's correlation) analyses were performed when appropriate. Odds ratios (OR) and $95 \%$ confidence interval (95\% CI) were calculated by standard formulas. To determine whether the association of CAD 
and hyperhomocystinemia was modulated by any other variable, we used multiple logistic regression analysis.

\section{Results}

A total of 236 patients had undergone angiography. Most were referred for a coronary angiogram for coronary disease investigation (83.9\%). Heart failure was the reason for the angiographic study in $6.8 \%$, valve disease in $6.3 \%$ and other reasons in $3 \%$ of cases. Only 13 patients $(5.5 \%)$ had no risk factor for CAD. One risk factor was present in 72 patients (30.5\%), while $86(36.5 \%)$ had two risk factors, $56(23.7 \%)$ had three, and 9 (9.8\%) had all of them.

Coronary angiograms showed 88 subjects with no stenotic lesion $>40 \%$, a result considered to indicate normal or near-nor-

\begin{tabular}{|c|c|c|c|}
\hline Characteristics & $\begin{array}{c}\text { CAD } \\
(N=148)\end{array}$ & $\begin{array}{l}\text { Normal or near-normal } \\
\text { arteries }(\mathrm{N}=88)\end{array}$ & $P$ \\
\hline \multicolumn{4}{|l|}{ Clinical } \\
\hline Age (years) & $58.6(10.9)$ & $54.7(12)$ & 0.01 \\
\hline Male sex & $98(66 \%)$ & 37 (42\%) & $<0.01$ \\
\hline Caucasian & $101(79 \%)$ & $58(66 \%)$ & 0.02 \\
\hline BMI $\left(\mathrm{kg} / \mathrm{m}^{2}\right)$ & $28.4(5)$ & $27.5(5)$ & ns \\
\hline Normal & $32(22 \%)$ & $27(30.7 \%)$ & ns \\
\hline Overweight & $64(43 \%)$ & $21(23.7 \%)$ & $<0.01$ \\
\hline Obese & $43(29 \%)$ & $28(31.8 \%)$ & ns \\
\hline Hyperlipidemia & $101(68 \%)$ & $33(37.5 \%)$ & $<0.001$ \\
\hline Diabetes & $53(36 \%)$ & $17(19 \%)$ & 0.01 \\
\hline Smoking & $45(30 \%)$ & $17(19 \%)$ & 0.06 \\
\hline Hypertension & $109(74 \%)$ & $73(83 \%)$ & ns \\
\hline \multicolumn{4}{|l|}{ Biochemical } \\
\hline Cholesterol (mg/dL) & $220.8(50.7)$ & $204.3(36.1)$ & 0.01 \\
\hline $\mathrm{HDL}(\mathrm{mg} / \mathrm{dL})$ & $36.0(11)$ & $42.9(13.5)$ & $<0.01$ \\
\hline LDL (mg/dL) & $150.9(45.6)$ & $132.2(31.9)$ & $<0.01$ \\
\hline Triglycerides (mg/dL) & $169.3(89.9)$ & $152.0(88.1)$ & ns \\
\hline Glucose (mg/dL) & $124.4(56.7)$ & $107.1(42.7)$ & 0.01 \\
\hline \multicolumn{4}{|l|}{ Nutritional } \\
\hline Vitamin B12 (pg/ml) & 488.3 (399) & $434(287)$ & ns \\
\hline Serum folate $(\mathrm{ng} / \mathrm{dL})$ & $7.4(3.3)$ & $7.7(3.6)$ & ns \\
\hline
\end{tabular}

Data are reported as means (SD) for continuous variables and numbers (\%) for categorical variables, and were analyzed statistically by 2 -sided $t$-test and chi-square test, respectively. $\mathrm{ns}=$ nonsignificant. mal arteries. One hundred and forty-eight patients $(62.7 \%)$ had coronary disease (at least one stenotic lesion $>40 \%$ in a major epicardic coronary).

\section{Characteristics of the patients}

Patients with CAD were older than those without significant coronary abnormalities (58.6 vs 54.7 years; $\mathrm{P}=0.01$ ), and were predominantly males. Body mass index was similar in both groups, despite a higher prevalence of overweight subjects among CAD patients. The prevalence of traditional risk factors was different in the two groups; hyperlipidemia was found in $68 \%$ of patients with CAD and in $37.5 \%$ of those without CAD $(\mathrm{P}<0.001)$. There was also a significant difference regarding diabetes (36 vs $19 \% ; \mathrm{P}=0.01$ ) and a trend toward a higher frequency of smoking among patients with CAD. No difference was observed regarding hypertension.

In relation to biochemical characteristics, plasma levels of total cholesterol and LDL-cholesterol were higher in patients with $\mathrm{CAD}$, whereas the inverse relation was seen with HDL-cholesterol levels. Plasma glucose levels were also higher in patients with CAD. No statistically significant difference was found in vitamin $B_{12}$ or plasma folate levels between groups. Clinical and biochemical features are listed in Table 1.

\section{Homocysteine}

Mean plasma Hcy level and prevalence of hyperhomocystinemia were higher in patients with CAD compared to those with normal or near-normal arteries: Hcy level [mean (SD)] was $14.4(6.8)$ vs $12.5(4.0) \mu \mathrm{M}$ $(\mathrm{P}=0.04)$ and hyperhomocystinemia was found in 31.1 vs $12.2 \%(\mathrm{P}=0.02)$.

By univariate analysis, the OR for coronary disease was $2.55(95 \% \mathrm{CI}=1.15-5.67)$ in patients with hyperhomocystinemia relative to subjects with plasma Hcy level below 
$17.3 \mu \mathrm{M}$. In a stepwise multivariate regression analysis, where age, creatinine, gender, hyperlipidemia, diabetes, and tobacco use were included, an independent association between hyperhomocystinemia and $\mathrm{CAD}$ was found $(\mathrm{OR}=2.48 ; 95 \% \mathrm{CI}=1.002$ 6.14). Age, male sex, hyperlipidemia, and diabetes were also independently associated with CAD (Table 2).

When plasma Hcy level was evaluated according to Friesinger score, we found a trend toward more advanced CAD in patients with hyperhomocystinemia. Patients with no arterial wall abnormalities (score 0 ) had the lowest mean Hcy level: $12 \pm 3.6 \mu \mathrm{M}$. Those with a score between 1 and 5 had 13.8 $\pm 5.3 \mu \mathrm{M}$, and those with a score of 6 to 10 had $14.1 \pm 7.4 \mu \mathrm{M}$. The highest mean plasma Hcy level was found in patients with the more severe CAD (scores 11 to 15): $14.5 \pm$ $7.1 \mu \mathrm{M}(\mathrm{P}=\mathrm{ns}$ by ANOVA). The difference between the two extreme groups was statistically significant $(P=0.04)$. The same trend was found regarding the prevalence of hyperhomocystinemia. Among patients with normal arteries (score 0 ), only $6 \%$ had hyperhomocystinemia. This proportion increased in patients with more extensive disease: $18 \%$ in patients with a score of 1 to 5 , $21 \%$ in patients with a score of 6 to 10 , and $23.5 \%$ in patients with a score of 11 to 15 (P $=0.19$ between all groups; $\mathrm{P}=0.03$ between patients with score 0 and score 11-15).

\section{Homocysteine, MTHFR polymorphism and folate status}

The C677T MTHFR polymorphism was found in the heterozygous form (CT genotype) in $30.9 \%$ of the study population, while 9.1\% had the homozygous form (TT genotype). The same proportion was found despite gender and race. We found no difference in TT genotype prevalence between patients with CAD and those without disease (data not shown).

Hcy level was similar regardless of
MTHFR genotype. Mean concentration was 12.3 (4.6) $\mu \mathrm{M}$ in patients with the $\mathrm{CC}$ genotype, $15.6(8.8) \mu \mathrm{M}$ in patients with the CT genotype, and 12.8 (4.7) $\mu \mathrm{M}$ in patients with the TT genotype $(\mathrm{P}=\mathrm{ns})$. Nevertheless, patients with the mutant allele had a higher prevalence of hyperhomocystinemia: Hcy above $17.3 \mu \mathrm{M}$ was present in $29 \%$ of homozygous individuals (TT), while this prevalence was $26 \%$ in patients with genotype CT and only $11 \%$ in the group with the wild genotype (genotype CC); $\mathrm{P}=0.03$.

Regarding nutritional factors, we found opposite results regarding vitamin $\mathrm{B}_{12}$ and folate. There was no significant correlation between Hcy and plasma vitamin $B_{12}$ levels $(\mathrm{r}=-0.035 ; \mathrm{P}=\mathrm{ns})$, but we found a negative and significant correlation with folate $(\mathrm{r}=$ $-0.14 ; \mathrm{P}=0.04)$. Comparing patients according to folate quartiles, we found that those with the lowest folate levels (1st quartile, folate $<4.9 \mathrm{ng} / \mathrm{dL}$ ) had a higher prevalence of hyperhomocystinemia than those in the highest quartile (folate $>9.4 \mathrm{ng} / \mathrm{dL})(30.3 \mathrm{vs}$ 9.2\%; $\mathrm{P}<0.01)$.

The correlation between Hcy and folate status differed according to MTHFR genotype (Table 3). In patients without the mutant allele, this correlation was not statistically significant $(\mathrm{r}=-0.11 ; \mathrm{P}=\mathrm{ns})$. However, in patients with genotypes CT and TT combined, a statistically significant correlation was demonstrable $(\mathrm{r}=-0.25 ; \mathrm{P}<0.05)$. The wild genotype seems to exert a protec-

Table 2. Variables independently associated with coronary artery disease (CAD) after stepwise multiple regression analysis.

\begin{tabular}{lcc}
\hline Variable & $\begin{array}{c}\text { Univariate analysis } \\
\text { OR }(95 \% \mathrm{Cl})\end{array}$ & $\begin{array}{c}\text { Multivariate analysis } \\
\text { OR }(95 \% \mathrm{Cl})\end{array}$ \\
\hline Gender (male) & $2.6(1.5 ; 4.6)$ & $3.86(1.97 ; 7.56)$ \\
Hyperlipidemia & $3.6(2.1 ; 6.2)$ & $3.96(2.08 ; 7.55)$ \\
Hyperhomocystinemia & $2.4(1.1 ; 5.4)$ & $2.48(1.002 ; 6.14)$ \\
Age & - & $1.04(1.01 ; 1.07)$ \\
Diabetes & $2.3(1.2 ; 4.4)$ & $2.14(1.02 ; 4.51)$
\end{tabular}

Multivariate models adjusted for age, gender, diabetes, smoking, serum creatinine, and hyperlipidemia. OR = odds ratio; $95 \% \mathrm{Cl}=95 \%$ confidence interval. 
tive effect against hyperhomocystinemia even in patients in the lowest folate quartile. In the same way, patients in the top folate quartile had low Hcy levels even when they carried the mutant allele.

\section{Additional factors related to homocysteine}

Men have had higher Hcy levels than women (14.9 (6.6) vs 12.4 (5.6) $\mu \mathrm{M}$; P < 0.001). Age was analyzed in two distinct ways, i.e., as a continuous and categorical variable ( $\leq 60$ or $>60$ years old). There was no relation between Hcy level and age in neither way, although patients with hyperhomocystinemia were older than those with Hcy $<17.3 \mu \mathrm{M}$ (60 vs 56 years; $\mathrm{P}=0.04)$.

Smoking, frequently related to highplasma Hcy levels, was not a cause of hyperhomocystinemia in our population. Homocysteine was 13.7 (5.0) $\mu \mathrm{M}$ in smokers and 13.8 (6.7) $\mu \mathrm{M}$ in non-smokers. Despite the fact that no patient with creatinine above $1.5 \mathrm{mg} / \mathrm{dL}$ was included in the study, we found a consistent and positive correlation between creatinine and Hcy $(\mathrm{r}=0.42 ; \mathrm{P}<$ $0.001)$.

\section{Discussion}

This is the first study from a Latin American country to show an independent association between hyperhomocystinemia and CAD. Our results are in agreement with the meta-analysis by Christen et al. (16), in which 43 studies were analyzed. In another metaanalysis, Boushley et al. (17) evaluated 27 studies and showed that Hcy is an independent and gradual risk factor for CAD. The OR for CAD ranged from 1.6 for men to 1.8 for women, for each Hcy level elevation of 5 $\mu \mathrm{M}$. The increase in coronary risk resulting from this increase is similar to an increase of $20 \mathrm{mg} / \mathrm{dL}$ in total cholesterol levels. The authors considered that $10 \%$ of the risk of the general population for the development of CAD can be attributed to Hcy.

Prospective studies reported conflicting results. Some identified Hcy as an independent risk factor $(5,18)$, but others did not $(19,20)$. Recently, Wald et al. (21) published a comprehensive meta-analysis of 16 prospective studies. Data from 144,936 patients with 3144 combined events were analyzed. The OR for a $5 \mu \mathrm{M}$ increase in Hcy level was

Table 3. Comparison between patients with poor (bottom quartile: $\leq 4.9 \mathrm{ng} / \mathrm{dL}$ ) and high (top quartile) folate status in terms of mean plasma homocysteine (Mean Hcy) levels and frequency of hyperhomocystinemia (Hyper Hcy) according to MTHFR genotype.

\begin{tabular}{|c|c|c|c|c|c|}
\hline & \multicolumn{4}{|c|}{ Folate quartiles } & \multirow[t]{2}{*}{$\mathrm{P}$} \\
\hline & 1st $(N=56)$ & 2nd $(N=58)$ & $3 r d(N=55)$ & 4th $(N=54)$ & \\
\hline \multicolumn{6}{|l|}{ All patients } \\
\hline Mean Hcy $(\mu \mathrm{M})$ & $15.7 \pm 7.5$ & $14.2 \pm 8.0$ & $12.5 \pm 3.7$ & $12.5 \pm 4.3$ & 0.05 \\
\hline Hyper Hcy & $30.3 \%$ & $20.0 \%$ & $9.1 \%$ & $9.2 \%$ & $<0.01$ \\
\hline \multicolumn{6}{|c|}{ CC genotype (60\% patients) } \\
\hline Mean Hcy $(\mu \mathrm{M})$ & $13.4 \pm 5.1$ & $13.0 \pm 4.7$ & $11.2 \pm 4.4$ & $12.3 \pm 4.1$ & ns \\
\hline Hyper Hcy & $18.5 \%$ & $15.9 \%$ & $14.8 \%$ & $10.7 \%$ & ns \\
\hline \multicolumn{6}{|c|}{ CT $(30.9 \%$ patients $)+$ TT (9.1\% patients) genotype } \\
\hline Mean Hcy $(\mu \mathrm{M})$ & $16.7 \pm 7.7$ & $17.4 \pm 12.2$ & $13.4 \pm 3.1$ & $11.8 \pm 4.3$ & 0.04 \\
\hline Hyper Hcy & $35.7 \%$ & $33.3 \%$ & $10.5 \%$ & $7.1 \%$ & ns \\
\hline
\end{tabular}

Data are reported as means $\pm \mathrm{SD}$ in $\mu \mathrm{M}$ for Mean Hcy and as percent for Hyper Hcy. Folate quartiles are: 1 st $\leq 4.9 \mathrm{ng} / \mathrm{dL}$, 2nd 4.9-7.0 ng/dL, 3rd 7.0-9.4 ng/dL, 4th >9.4 ng/dL. P. Bottom vs top quartile. Mean Hcy level compared by $t$-test after log transformation; frequency (\%) compared by chi-square test. $n s=$ nonsignificant. 
$1.23(95 \% \mathrm{CI}=1.14-1.32)$. Zylberstein et al. (22) published the results of a 24-year follow-up evaluating Hcy as a risk factor for coronary morbidity and mortality in 1368 women. For the fifth Hcy quintile, relative risk was 1.86 (95\% CI $=1.06$ to 3.26 ) for acute myocardial infarction and 5.14 (95\% $\mathrm{CI}=2.22$ to 11.92 ) for death due to acute myocardial infarction.

Toole et al. (23) analyzed the effect of homocysteine-lowering therapy upon recurrent stroke. Despite persistent association between baseline Hcy levels and outcome, folate, vitamins $\mathrm{B}_{12}$ and $\mathrm{B}_{6}$ alone or in combination did not prevent new vascular event after 2 years. However, this and other studies had their statistical power questioned (24). Also, the mandatory fortification of food with folate in certain countries has masked the effect of treatment on stroke risk (25).

Only a few studies have assessed the relationship between plasma Hcy levels and the extent of coronary disease. In 70 patients, Tsai et al. (26) demonstrated a positive correlation between Hcy levels and the extent of coronary disease, but clearly only in patients with a low CAD risk profile (less than 3 risk factors). Evaluating only the number of injured vessels, Chao et al. (27) showed that patients with progressively higher Hcy levels have more arteries injured. The Friesinger score that we used is not only accurate for evaluation of extension and severity of coronary disease, but also as a prognostic indicator. In the CASS study (28), its prognostic value was better than scores that considered only the number of injured vessels or coronaries with proximal stenotic lesions.

The mechanism(s) by which Hcy is atherogenic is still not completely understood. Endothelial dysfunction seems to play a major role. Hcy is a highly reactive amino acid that produces endothelial injury in both experimental animals and cell cultures (30).

Woo et al. (31) demonstrated impaired flow-mediated dilatation of the brachial artery in 17 healthy individuals who had no other risk factor except hyperhomocystinemia; furthermore, Hcy levels and endothelial function were corrected with folic acid supplementation. Since toxicity is dependent on the degree of hyperhomocystinemia, this could explain, at least in part, the graded effect we found between Hcy concentration and the extent of CAD.

The inverse and significant correlation of Hcy with folate, but not with vitamin $B_{12}$, are compatible with the metabolic pathway of Hcy. Folate is a substrate in the remethylation cycle, where Hcy is converted to methionine, in which it donates the methyl group for this reaction. On the other hand, vitamins $\mathrm{B}_{12}, \mathrm{~B}_{2}$ and $\mathrm{B}_{6}$ function only as enzyme cofactors. Although median folate level was similar and within the normal range in both patients and controls, a considerable proportion of patients had low-plasma folate levels. The lower limit proposed by the WHO is 6 $\mathrm{ng} / \mathrm{mL}$. Almost $30 \%$ of patients had folate below this limit, and their Hcy levels were higher than those of patients with folate within the normal range. Larger studies have previously shown this relation. In the Framinghan study (32), patients in the lowest decile of folate had mean Hcy $15.6 \mu \mathrm{M}$, while those in the superior decile had $11 \mu \mathrm{M}$.

The data regarding the prevalence of C677T MTHFR polymorphism showed that the TT genotype occurs in our population at a similar frequency as that observed in Caucasian populations (33). Arruda et al. (34), evaluated the C677T polymorphism in three distinct ethnic groups in Brazil. Genotype TT was found in a similar prevalence among Caucasian descent (10\%). The prevalence was lower among Black $(1.45 \%)$ and Indian populations $(1.2 \%)$.

We also assessed the combined effect of low plasma folate levels in patients with MTHFR polymorphism. As previously mentioned, $40 \%$ of the population carries the mutant allele either in homozygosis $(9.1 \%)$ 
or heterozygosis (30.9\%). This group is particularly susceptible to hyperhomocystinemia when exposed to low folate levels. Accordingly, countries such as the United States and Canada already enrich their grains with folic acid in an effort to reduce folate-neural tube defects. If lowering Hcy levels is proved to reduce cardiovascular risk, a high folate intake should be recommended for everyone. On the other hand, patients with the wild genotype (CC) seem to be less prone to increased Hcy levels, even in the presence of low folate levels.
Our findings are relevant since several studies have shown that Hcy levels differ among countries with similar nutritional habits. Thus, it may be useful to know the data for our population. Alfthan et al. (35) have demonstrated these inter-country differences among several European countries, Japan and Israel. Moreover, these investigators demonstrated a direct relationship between plasma Hcy concentration and mortality from all cardiovascular diseases according to WHO data.

\section{References}

1. McCully KS (1969). Vascular pathology of homocysteinemia: implications for the pathogenesis of arteriosclerosis. American Journal of Pathology, 56: 111-128.

2. Bolander-Gouaille C (2002). Focus on Homocysteine and the Vitamins Involved in its Metabolism. Springer Verlag, Paris, France, 15.

3. Harker CA, Slichter SF, Scott CR et al. (1974). Homocysteinemia: vascular injury and arterial thrombosis. New England Journal of Medicine, 291: 537-543.

4. Clarke R, Daly L, Robinson K et al. (1991). Hyperhomocysteinemia: an independent risk factor for vascular disease. New England Journal of Medicine, 324: 1149-1155.

5. Stampfer MJ, Malinow MR, Willett WC et al. (1992). A prospective study of plasma homocyst(e)-ine and risk of myocardial infarction in US physicians. Journal of the American Medical Association, 268: 877-881.

6. Shai I, Stampfer MJ, Ma J et al. (2004). Homocysteine as a risk factor for coronary heart diseases and its association with inflammatory biomarkers, lipids and dietary factors. Atherosclerosis, 177: 375-381.

7. Perry IJ, Refsum H \& Morris RW (1995). Prospective study of serum total homocysteine concentration and risk of stroke in middle-aged British men. Lancet, 346: 1395-1398.

8. Boers GH, Smals AG, Trijbels FJ et al. (1985). Heterozygosity for homocystinuria in premature peripheral and cerebral occlusive arterial disease. New England Journal of Medicine, 313: 709-715.

9. Homocysteine Lowering Trialists' Collaboration (1998). Lowering blood homocysteine with folic acid based supplements: meta-analysis of randomized trials. British Medical Journal, 316: 894-898.

10. Rozen R (1977). Genetic predisposition to hyperhomocystinemia: deficiency of methylenetetrahydrofolate reductase (MTHFR). Journal of Thrombosis and Haemostasis, 78: 523-526.

11. United Nations/Statistics Division: World and regional trends: data for years around 1990 and $2000 \mathrm{http} / \mathrm{unstats}$.un.org/unsd/mi/ mi_wrtrends1.asp.

12. Malaquias Filho M \& Rissin A (2003). Nutritional transition in Brazil: geographic and temporal trends. Cadernos de Saúde Pública, 19 (Suppl I): S181-S191.
13. Friesinger GC, Page EE \& Ross RS (1970). Prognostic significance of coronary arteriography. Transactions of the Association of American Physicians, 83: 78-92.

14. Fiskerstrand T, Refsum H, Kvalheim G et al. (1993). Homocysteine and other thiols in plasma and urine: automated determination and sample stability. Clinical Chemistry, 39: 263-271.

15. Bravo-Osorio M \& BydLowski SP (2000). Detection of methylenetetrahydrofolate reductase (MTHFR) C677T and prothrombin G20210A mutations: second restriction site for digestion control of PCR products. Clinica Chimica Acta, 301: 219-223.

16. Christen WG, Ajani UA, Glynn RJ et al. (2000). Blood levels of homocysteine and increased risks of cardiovascular disease: causal or casual? Archives of Internal Medicine, 160: 422-434.

17. Boushley CJ, Beresford SAA, Omenn GS et al. (1995). A quantitative assessment of plasma homocysteine as a risk factor for vascular disease: probable benefits of increasing folic acid intakes. Journal of the American Medical Association, 274: 1049-1057.

18. Arnesen E, Refsum H \& Bonaa KH (1995). Serum total homocysteine and coronary heart disease. International Journal of Epidemiology, 24: 704-709.

19. Evans RW, Shaten BJ, Hempel JD et al. (1997). Homocyst(e)ine and risk of cardiovascular disease in the Multiple Risk Factor Intervention Trial. Arteriosclerosis, Thrombosis, and Vascular Biology, 17: 1947-1953.

20. Folsom AR, Nieto FJ, McGovern PG et al. (1998). Prospective study of coronary heart disease incidence in relation to fasting total homocysteine, related genetic polymorphisms, and B vitamins. The Atherosclerosis Risk in Communities (ARIC) study. Circulation, 98: 204-210.

21. Wald DS, Law M \& Morris JK (2002). Homocysteine and cardiovascular disease: evidence on causality from a meta-analysis. British Medical Journal, 325: 1202.

22. Zylberstein DE, Bengtsson C, Bjorkelund C et al. (2004). Serum homocysteine in relation to mortality and morbidity from coronary heart disease: a 24-year follow-up of the population study of women in Gothenburg. Circulation, 109: 601-606.

23. Toole JF, Malinow MR, Chambless LE et al. (2004). Lowering ho- 
mocysteine in patients with ischemic stroke to prevent recurrent stroke, myocardial infarction, and death. The Vitamin Intervention for Stroke Prevention (VISP) randomized controlled trial. Journal of the American Medical Association, 291: 565-575.

24. Bostom AG, Selhub J, Jacques PF et al. (2001). Power shortage: clinical trials testing the "Homocysteine Hypothesis" against a background of folic acid-fortified cereal grain flour. Annals of Internal Medicine, 135: 133-137.

25. Schwammenthal Y \& Tanne D (2004). Homocysteine, B-vitamin supplementation, and stroke prevention: from observational to interventional trials. Lancet Neurology, 3: 493-495.

26. Tsai WC, Li YH, Tsai LM et al. (2000). Correlation of homocysteine levels with the extent of coronary atherosclerosis in patients with low cardiovascular risk profiles. American Journal of Cardiology, 85: 4952.

27. Chao CL, Tsai HH, Lee CM et al. (1999). The graded effect of hyperhomocystinemia on the severity and extent of coronary atherosclerosis. Atherosclerosis, 147: 379-386.

28. Ringqvist I, Fisher LD, Mock M et al. (1983). Prognostic value of angiographic indices of coronary artery disease from the Coronary Artery Surgery Study (CASS). Journal of Clinical Investigation, 71: 1854-1866.
29. Ray JG (1998). Meta-analysis of hyperhomocystinemia as a risk factor for venous thromboembolic disease. Archives of Internal Medicine, 158: 2101-2106.

30. Wall RT, Harlan JM, Harker LA et al. (1980). Homocysteine-induced endothelial cell injury in vitro: a model for the study of vascular injury. Thrombosis Research, 18: 113-121.

31. Woo KS, Chook P \& Lolin YI (1999). Folic acid improves arterial endothelial function in adults with hyperhomocystinemia. Journal of the American College of Cardiology, 34: 2002-2006.

32. Selhub J, Jacques PF, Wilson PW et al. (1993). Vitamin status and intake as primary determinants of homocysteinemia in an elderly population. Journal of the American Medical Association, 270: 26932698.

33. Brattstrom L, Zhang Y, Hurtig M et al. (1998). A common methylenetetrahydrofolate reductase gene mutation and longevity. Atherosclerosis, 141: 315-319.

34. Arruda VR, Siqueira LH, Goncalves MS et al. (1998). Prevalence of the mutation $\mathrm{C} 677 \rightarrow \mathrm{T}$ in the methylenetetrahydrofolate reductase gene among distinct ethnic groups in Brazil. American Journal of Medical Genetics, 78: 332-335.

35. Alfthan G, Aro A \& Gey KF (1997). Plasma homocysteine and cardiovascular disease mortality. Lancet, 349: 397. 